\title{
Numerical simulations of the cosmic star formation history
}

\author{
Y. Ascasibar ${ }^{1,2}$, G. Yepes ${ }^{1}$, S. Gottlöber ${ }^{2}$, and V. Müller ${ }^{2}$ \\ 1 Grupo de Astrofísica, C-XI Universidad Autónoma de Madrid, 28049 Madrid, Spain \\ 2 Astrophysikalisches Institut Potsdam, An der Sternwarte 16, 14482, Germany
}

Received 12 November 2001 / Accepted 27 February 2002

\begin{abstract}
The cosmic star formation history in Cold Dark Matter dominated cosmological scenarios is studied by means of hydrodynamical numerical simulations. In particular, we explore a low density model with a $\Lambda$-term and two high density models with different power spectra, all of them being spatially flat. Our simulations employ a fully nonlinear $N$-body and Eulerian hydrodynamics algorithm with a model for star formation and supernovae feedback that depends on two phenomenological parameters determined in agreement with previous papers. We find a nearly constant star formation rate beyond $z=1$, and we discuss which facts may determine the decrease in the SFR from $z=1$ to the present epoch. The $\Lambda$-term cosmology with realistic parameters for star formation and feedback best reproduces the observed star formation history.
\end{abstract}

Key words. galaxies: formation, stellar content - cosmology: observations, theory - stars: formation methods: $N$-body simulations

\section{Introduction}

A detailed understanding of galaxy formation remains one of the primary goals of modern cosmology. Whereas gravitation is basically responsible for the large scale structure of the universe, many physical phenomena, covering a broad dynamical range, are involved on galactic scales. A reliable treatment of baryonic physics is essential in order to obtain a consistent picture of galaxy formation and evolution, where star formation and feedback to the interstellar medium (ISM) play a key role.

The star formation history of the universe, i.e. the global star formation rate (SFR) density as a function of redshift, is a crucial test for galaxy formation scenarios. Many measurements of this quantity have been undertaken during the past few years, and the number and accuracy of available observations are still rapidly increasing.

Photometric redshift estimates based on Lyman-limit systems (Steidel et al. 1996a, 1996b) stimulated the study of the rest frame UV continuum flux around $2000 \AA$ as a preferred wavelength range for objects in the far universe. This emission traces the presence of massive (and therefore young) stars and can be related directly to the actual star formation rate. It is relatively easy to detect at high $z$ as it is redshifted into the optical band. However, older stellar populations and AGN can make a significant contribution as well, leading to an overestimation of the total SFR. On the other hand, dust enshrouding star formation

Send offprint requests to: Y. Ascasibar,

e-mail: yago.ascasibar@uam.es regions absorb very efficiently the UV light, re-emitting it in the far IR. Unfortunately, there is still some uncertainty to account for dust extinction and its evolution with redshift (Nagamine et al. 2001).

Recombination lines from H II regions can be in principle a more reliable estimator of the instantaneous SFR, since the ionizing radiation $(\lambda \leq 912 \AA)$ comes from more massive (younger) stars than the softer UV continuum. These optical lines are less dramatically affected by dust extinction and can be mapped with higher resolution in the local universe, but they require infrared spectroscopy at moderate redshifts, which has not been possible until very recently due to the faintness of the sources involved (Pettini et al. 1998). For $\mathrm{H}_{\alpha}$, this happens at $z \sim 0.5$, and for forbidden lines, such as $[\mathrm{O}$ II $] 3727 \AA$, at $z \sim 1.5$. There is also a considerable degree of uncertainty in the relation between recombination lines and star formation activity.

Finally, the far infrared (FIR) spectrum of a galaxy can be roughly separated into two components, namely the thermal emission of dust (heated by star formation bursts) around $\lambda \sim 60 \mu \mathrm{m}$ and an infrared cirrus powered by the global radiation field, which dominates for $\lambda \geq 100 \mu \mathrm{m}$. Neglecting cirrus and AGN contamination, the FIR luminosity would also be an excellent tracer, but instrumentation is not as developed in the IR and radio bands as it is in the optical, and the conversion factor between FIR luminosity and SFR is for the moment rather model dependent.

Although there is a large scatter among different indicators at any given redshift, basically all studies find a 
significant increase (by about one decade) in the cosmic $\mathrm{SFR}$ density from the present day to $z=1$. However, it is still a controversial issue (Hopkins et al. 2001) whether it reaches a broad maximum there (Madau et al. 1996; Gispert et al. 2000) or it declines gradually towards higher redshifts (Sawicki et al. 1997; Pascarelle et al. 1998; Steidel et al. 1999).

From a theoretical point of view, star formation is a highly non-linear process, which precludes any simple analytical treatment. Most efforts towards modeling the star formation history of the universe resort to semi-analytical methods or numerical simulations to tackle the feedback mechanisms that self-regulate the SFR within the hierarchical clustering scenario of structure formation.

Semi-analytical models (Kauffmann et al. 1993; Cole et al. 1994) construct a large sample of Monte Carlo realizations of halo merging histories, using the PressSchechter formalism. Hydrodynamics, star formation and feedback are implemented through simple recipes, whose parameters are fixed in order to match the observed properties of real galaxies, specially the luminosity function and the Tully-Fisher relation (Somerville \& Primack 1999). Their high computational efficiency allows a fast exploration of the parameter space in search of a viable model, but the number of free parameters asks for supplementary modeling based on more fundamental physics.

Numerical simulations are intended to solve directly the set of differential equations of gravitation and gas hydrodynamics, and hence require much fewer model assumptions than the semi-analytic approach. However, despite the staggering advances in computer technology and numerical algorithms, resolution in large-scale hydrodynamical simulations is severely restricted by computational and data storage requirements. Although gas can be treated self-consistently, some phenomenological recipes are still required in order to model star formation and feedback.

The cosmic SFR density is a direct outcome of semianalytical methods (Somerville et al. 2001) and hydrodynamical simulations (Nagamine et al. 2001). Both of them predict a significant star formation activity at high $z$, favoring the hypothesis of a gradual evolution of the comoving SFR as a function of time.

The present analysis is based on a series of numerical simulations, within different cosmological models. In previous papers, we have studied basic observable properties of simulated galaxy samples, such as the luminosity function and color dependence on environment (Elizondo et al. 1999a), and the Tully-Fisher relation (Elizondo et al. 1999b). In this paper, we will focus on the evolution of the SFR on a cosmological scale, comparing our results with recent observations.

The following section is devoted to the observational estimation of the cosmic SFR. The standard procedure is briefly summarized and a compilation of recent results is included with an analytical prescription for the transformation between cosmological models. Section 3 describes the code and the different numerical experiments on which the present paper is based. Our results are presented in Sect. 4, where we compare with observations and evaluate the separate contribution of cosmology, feedback effects and environment. Our main conclusions are summarized in Sect. 5.

\section{Observations}

The first step to obtain an estimate of the SFR density from observations is the selection of a complete sample of galaxies at a given redshift and wavelength. Some authors (Steidel et al. 1999) claim that the HDF covers a very small area $\left(\sim 5 \operatorname{arcmin}^{2}\right)$, and hence it is not statistically representative of the whole universe. This cosmic variance is difficult to quantify, but the strong clustering of high redshift galaxies (and the fact that most estimates beyond $z=2$ are based on the HDF) seems to indicate that it could be an important effect.

Another crucial issue is the correction for incompleteness in the construction of the luminosity function, which can be done following the $V_{\max }$ formalism (Schmidt 1968) or resorting to more elaborate Monte Carlo algorithms (e.g. Steidel et al. 1999). The comoving luminosity density is easily obtained by integration over all magnitudes (extrapolating the faint end as a Schechter function), and then it can be converted to a SFR density with the help of a population synthesis model.

A compilation of recent results is given in Table 1. The first column indicates the bibliographic reference, as well as the survey from which the galaxy sample was extracted. Next columns correspond to the chosen wavelength and redshifts of each analysis, and finally the comoving luminosity and SFR densities.

Conversion factors between the last two quantities differ significantly from author to author, and in some cases only the luminosity density was provided. If there is a SFR estimate in the original paper, then it is quoted in Table 1 corrected for dust extinction; else, we follow Kennicutt's (1998) prescription for an exponential burst and a Salpeter IMF between 0.1 and $100 M_{\odot}$ :

$\dot{\rho}_{*}\left[M_{\odot} \mathrm{yr}^{-1}\right]=\left\{\begin{array}{l}1.4 \times 10^{-28} L_{\mathrm{UV}}\left[\mathrm{erg} \mathrm{s}^{-1} \mathrm{~Hz}^{-1}\right] \\ 1.4 \times 10^{-41} L_{3727}\left[\mathrm{erg} \mathrm{s}^{-1} \mathrm{~Hz}^{-1}\right] .\end{array}\right.$

For dust extinction, we have applied a correction of $A(1500-2000 \AA)=1.2 \mathrm{mag}$ and $A(2880 \AA, \mathrm{O}$ II $)=$ $0.625 \mathrm{mag}$, which correspond to factors of 3.02 and 1.78 respectively. All modifications to the original data are shown as a footnote on the bibliographic reference in Table 1.

Most observational values given in Table 1 were calculated assuming a standard cold dark matter (SCDM) cosmology with dimensionless Hubble parameter $h=0.5$ and mean matter density $\Omega_{\mathrm{m}}=1$. Since the conversion factor from luminosity to SFR and the correction for dust extinction are completely independent of the cosmological scenario, the computation of the comoving SFR density in a $\Lambda \mathrm{CDM}(h=0.7)$ model only involves the transformation of the luminosity densities. 
Table 1. Observational estimates of the cosmic SFR density at different epochs. $\rho_{\mathrm{L}}$ refers to the luminosity density at the appropriate wavelength, expressed in $\mathrm{erg} \mathrm{s}^{-1} \mathrm{~Hz}^{-1} \mathrm{Mpc}^{-3} \cdot \dot{\rho}_{*}$ represents the comoving SFR density (in $M_{\odot} \mathrm{yr}^{-1} \mathrm{Mpc}^{-3}$ ) for our SCDM and $\Lambda$ CDM cosmologies. ${ }^{\mathrm{d}}$ Original data has been corrected for dust extinction.

\begin{tabular}{|c|c|c|c|c|c|}
\hline SURVEY & EsTIMATOR & $\bar{z}$ & $\overline{l o g} \rho_{\mathrm{L}}^{\mathrm{SCDM}}$ & $\overline{l o g} \dot{\rho}_{*}^{\text {SCDM }}$ & $\overline{l o g} \dot{\rho}_{*}^{\Lambda \mathrm{CDM}}$ \\
\hline \multirow[t]{8}{*}{ HDF (Pascarelle et al. 1998) ${ }^{\mathrm{d}}$} & $1500 \AA$ & $0.25 \pm 0.25$ & $26.47_{-022}^{+0.31}$ & -1.38 & -1.36 \\
\hline & & $0.75 \pm 0.25$ & $26.61_{-0.14}^{+0.23}$ & -1.24 & -1.26 \\
\hline & & $1.25 \pm 0.25$ & $26.80_{-0.12}^{+0.14}$ & -1.05 & -1.10 \\
\hline & & $1.75 \pm 0.25$ & $26.83_{-0.12}^{+0.24}$ & -1.02 & -1.08 \\
\hline & & $2.50 \pm 0.50$ & $26.62_{-0.21}^{+0.28}$ & -1.23 & -1.30 \\
\hline & & $3.50 \pm 0.50$ & $26.59_{-0.27}^{+0.21}$ & -1.26 & -1.34 \\
\hline & & $4.50 \pm 0.50$ & $26.70_{-0.37}^{+0.27}$ & -1.15 & -1.23 \\
\hline & & $5.50 \pm 0.50$ & $26.34_{-0.38}^{+0.59}$ & -1.51 & -1.59 \\
\hline \multirow[t]{2}{*}{ HDF (Madau et al. 1998) d } & $1500 \AA$ & $2.75 \pm 0.75$ & $26.90 \pm 0.15$ & -0.95 & -1.02 \\
\hline & & $4.00 \pm 0.50$ & $26.50 \pm 0.20$ & -1.35 & -1.43 \\
\hline \multirow[t]{2}{*}{ (Steidel et al. 1999) } & $1700 \AA$ & $3.04 \pm 0.35$ & $27.04 \pm 0.07$ & -0.81 & -0.89 \\
\hline & & $4.13 \pm 0.35$ & $26.95 \pm 0.10$ & -0.90 & -0.98 \\
\hline$\left(\right.$ Treyer et al. 1998) ${ }^{\mathrm{d}}$ & $2000 \AA$ & $0.15 \pm 0.15$ & $26.45 \pm 0.15$ & -1.40 & -1.35 \\
\hline \multirow[t]{3}{*}{ CFRS (Lilly et al. 1996) d } & $2800 \AA$ & $0.35 \pm 0.15$ & $26.14 \pm 0.07$ & -1.71 & -1.68 \\
\hline & & $0.625 \pm 0.125$ & $26.46 \pm 0.08$ & -1.39 & -1.39 \\
\hline & & $0.875 \pm 0.125$ & $26.78 \pm 0.15$ & -1.07 & -1.10 \\
\hline \multirow[t]{4}{*}{ HDF (Cowie et al. 1999) ${ }^{d}$} & $2800 \AA$ & $0.35 \pm 0.15$ & $25.91 \pm 0.12$ & -1.69 & -1.66 \\
\hline & & $0.625 \pm 0.125$ & $26.15 \pm 0.12$ & -1.45 & -1.45 \\
\hline & & $0.875 \pm 0.125$ & $26.06 \pm 0.12$ & -1.54 & -1.57 \\
\hline & & $1.25 \pm 0.25$ & $26.35 \pm 0.10$ & -1.25 & -1.30 \\
\hline \multirow[t]{3}{*}{ HDF (Connolly et al. 1997) ${ }^{\mathrm{d}}$} & $2800 \AA$ & $0.75 \pm 0.25$ & $26.77 \pm 0.15$ & -1.08 & -1.10 \\
\hline & & $1.25 \pm 0.25$ & $26.94 \pm 0.15$ & -0.91 & -0.96 \\
\hline & & $1.75 \pm 0.25$ & $26.84 \pm 0.15$ & -1.26 & -1.32 \\
\hline \multirow[t]{5}{*}{ HDF (Sawicki et al. 1997) ${ }^{\mathrm{d}}$} & $3000 \AA$ & $0.35 \pm 0.15$ & $26.51 \pm 0.47$ & -1.34 & -1.31 \\
\hline & & $0.75 \pm 0.25$ & $26.74 \pm 0.06$ & -1.11 & -1.13 \\
\hline & & $1.50 \pm 0.50$ & $26.93 \pm 0.05$ & -0.92 & -0.97 \\
\hline & & $2.50 \pm 0.50$ & $27.28 \pm 0.06$ & -0.57 & -0.64 \\
\hline & & $3.50 \pm 0.50$ & $26.91 \pm 0.10$ & -0.94 & -1.02 \\
\hline (Gallego et al. 1995) & $\mathrm{H}_{\alpha}$ & $0.025 \pm 0.025$ & $39.09 \pm 0.04$ & -2.01 & -1.89 \\
\hline CFRS (Tresse \& Maddox 1998) & $\mathrm{H}_{\alpha}$ & $0.2 \pm 0.1$ & $39.44 \pm 0.04$ & -1.66 & -1.60 \\
\hline CFRS (Glazebrook et al. 1999) & $\mathrm{H}_{\alpha}$ & $0.875 \pm 0.125$ & $40.01 \pm 0.15$ & -0.91 & -0.94 \\
\hline (Yan et al. 1999) & $\mathrm{H}_{\alpha}$ & $1.3 \pm 0.5$ & $40.21 \pm 0.13$ & -0.89 & -0.94 \\
\hline \multirow[t]{3}{*}{ CFRS (Hammer et al. 1997) d } & O II $3727 \AA$ & $0.35 \pm 0.15$ & $38.63_{-0.08}^{+0.06}$ & -1.97 & -1.94 \\
\hline & & $0.625 \pm 0.125$ & $39.16_{-0.15}^{+0.08}$ & -1.44 & -1.44 \\
\hline & & $0.875 \pm 0.125$ & $39.56_{-0.38}^{+0.15}$ & -1.04 & -1.07 \\
\hline \multirow[t]{3}{*}{ CFRS (Flores et al. 1999) } & $15 \mu \mathrm{m}$ & $0.35 \pm 0.15$ & $41.97 \pm 0.25$ & -1.46 & -1.43 \\
\hline & & $0.625 \pm 0.125$ & $42.20 \pm 0.25$ & -1.15 & -1.15 \\
\hline & & $0.875 \pm 0.125$ & $42.53 \pm 0.25$ & -0.82 & -0.85 \\
\hline & $60 \mu \mathrm{m}$ & $3.0 \pm 1.0$ & $\leq 42.26$ & -0.98 & -1.05 \\
\hline \multirow{5}{*}{ (Haarsma et al. 2000) } & $1.4 \mathrm{GHz}$ & $0.28 \pm 0.12$ & $26.75_{-0.21}^{+0.14}$ & -1.17 & -1.13 \\
\hline & & $0.46 \pm 0.05$ & $27.03_{-0.21}^{+0.21}$ & -0.89 & -0.87 \\
\hline & & $0.60 \pm 0.05$ & $27.12_{-0.26}^{+0.16}$ & -0.80 & -0.80 \\
\hline & & $0.81 \pm 0.08$ & $27.39_{-0.18}^{+0.13}$ & -0.53 & -0.55 \\
\hline & & $1.60 \pm 0.64$ & $27.54_{-0.18}^{+0.18}$ & -0.38 & -0.44 \\
\hline
\end{tabular}

In a flat universe, the volume enclosed by a solid angle $\Delta \omega$ between $z-\Delta z$ and $z+\Delta z$ is

$V(z, \Delta z)=\frac{4 \pi}{3} \Delta \omega\left(d_{\mathrm{m}}^{3}(z+\Delta z)-d_{\mathrm{m}}^{3}(z-\Delta z)\right)$

where $d_{\mathrm{m}}(z)$ is the comoving distance in each cosmological model, while luminosity scales with luminosity distance as $d_{\mathrm{L}}(z)=(1+z) d_{\mathrm{m}}(z)$. Then, assuming that all galaxies are located at the centers of the interval in $z$, the luminosity density (and hence the total SFR density) will be proportional to

$\dot{\rho}_{*}(z) \propto \frac{L(z)}{V(z, \Delta z)} \propto \frac{d_{\mathrm{m}}^{2}(z)}{d_{\mathrm{m}}^{3}(z+\Delta z)-d_{\mathrm{m}}^{3}(z-\Delta z)}$.

To first order in $\Delta z$, the conversion between SFR densities in $\Lambda \mathrm{CDM}$ and variants of the SCDM $(h=0.5)$ cosmologies can be approximated as

$\frac{\dot{\rho}_{*}^{\Lambda \mathrm{CDM}}}{\dot{\rho}_{*}^{\mathrm{SCDM}}} \sim \frac{h^{\Lambda \mathrm{CDM}}}{h^{\mathrm{SCDM}}} \sqrt{\Omega_{\mathrm{m}}+\Omega_{\Lambda}(1+z)^{-3}}$. 


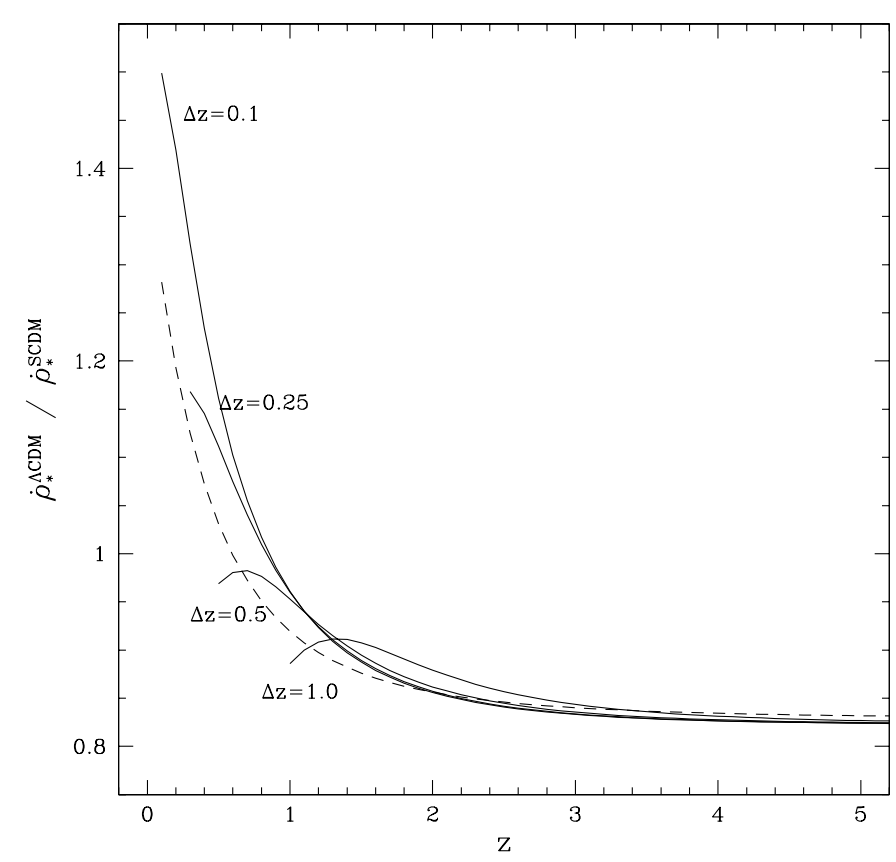

Fig. 1. Conversion factor between star formation densities in our cosmological models $\operatorname{SCDM}\left(h=0.5, \Omega_{\mathrm{m}}=1, \Omega_{\Lambda}=0\right)$ and $\Lambda \mathrm{CDM}\left(h=0.7, \Omega_{\mathrm{m}}=0.35, \Omega_{\Lambda}=0.65\right)$ for several redshift intervals $\Delta z$. Dashed line corresponds to the approximation (4).

Figure 1 shows this factor, as well as the exact expression for different values of $\Delta z$, which requires a numerical evaluation of the luminosity distance in the $\Lambda \mathrm{CDM}$ model. Although the results are very similar, we have chosen to use the exact expression (3) to compute the SFRs in Table 1 in order to minimize errors.

At low redshifts, the data are consistent for all the independent tracers of the star formation activity once the effect of dust obscuration is taken into account. The steep increase in the cosmic SFR density from the present day to $z \sim 1$ is firmly established. However, there is not yet a full agreement about whether there is a characteristic epoch of star formation in the universe around $z \sim 1.5$ or on the contrary that the SFR declines very slowly towards higher $z$, as more recent observations seem to point out. The results of ongoing deep surveys will be extremely helpful in order to reach a definitive conclusion from the observational point of view.

\section{Simulations}

\subsection{Cosmological models}

Up to now, the $\Lambda$ CDM model has proven to be very successful in describing most of the observational data at both low and high redshifts. Moreover, recent observations indicate the existence of a positive cosmological constant (Perlmutter et al. 1999; Melchiorri et al. 2000). For our study we have chosen a spatially flat universe with a cosmological constant $\Lambda / 3 H_{0}^{2} \equiv \Omega_{\Lambda}=0.65$ and a present-day Hubble constant of $H_{0}=100 \mathrm{~h}=70 \mathrm{~km} \mathrm{~s}^{-1} / \mathrm{Mpc}$. In order to study the influence of cosmology on the global SFR, we have investigated three other scenarios: $\Lambda \mathrm{CDM}$ with lower $\Omega_{\Lambda}=0.6$, the standard CDM model (dominated by dark matter density), and the BSI model with a Broken Scale Invariant perturbation spectrum (as predicted by double inflation, Gottlöber et al. 1991). The latter model is also dark matter-dominated, but it describes a primordial power spectrum in which fluctuations on small scales are significantly suppressed. It is almost the same spectrum as in the $\tau$ CDM model, in which the primordial perturbation spectrum has been changed due to the hypothetical decay of massive $\tau$ neutrinos (Efstathiou et al. 1992). The model parameters are summarized in Table 2. All spectra are COBE-normalized. The normalization is given by the linear dark matter mass variance $\sigma_{8}$ in a $8 h^{-1} \mathrm{Mpc}$ sphere extrapolated to present time.

\subsection{Numerical code}

The code used to perform the numerical experiments consists of a combination of a Particle-Mesh $N$-body gravity solver and an Eulerian Piecewise Parabolic Method to account for gasdynamics. A detailed modeling of the most relevant non-adiabatic baryonic processes (i.e. multiphase ISM, cooling, photoionization, star formation and supernovae feedbacks) acting at a sub-grid level is also incorporated. The code is described in detail elsewhere (Yepes et al. 1997). Here we briefly review its main features regarding star formation and feedback.

The ISM is modeled as a two phase medium: hot gas $\left(T_{\mathrm{h}}>10^{4} \mathrm{~K}\right)$ and a cold phase $\left(T_{\mathrm{c}}=10^{4} \mathrm{~K}\right)$, which pretends to mimic the molecular clouds from which stars are formed.

Cold clouds can be formed from the hot gas by radiative and Compton cooling or by thermal instability. Radiative cooling rates, $\Lambda_{\mathrm{r}}(T, Z)$, are taken from Sutherland \& Dopita (1993) for two metallicity cases: either primordial composition or solar, depending on the star formation history of each computational element.

Stars are created, from the cold phase, as collisionless particles at each timestep. Their mass is proportional to the density of cold gas in each cell:

$\dot{\rho}_{*}=-\dot{\rho}_{\mathrm{c}}=(1-\beta) \frac{\rho_{\mathrm{c}}}{t_{*}}$

where $\beta$ is the fraction of massive stars $\left(M>10 M_{\odot}\right)$ that explode as supernovae. For a Salpeter IMF, $\beta=0.12$. For the characteristic time of star formation $t_{*}$ we assume a constant value of $10^{8} \mathrm{yr}$.

Several feedback mechanisms are included: photoionisation by UV light, gas heating and evaporation by supernova explosions. Massive stars are assumed to create a homogeneously distributed background of ionizing radiation, and gas should be dense enough to screen it in order to be able to cool into the cold phase and form stars (e.g. Giroux \& Shapiro 1986; Mücket \& Kates 1997). A simple prescription, without resorting to a detailed computation of photoionization heating is to impose an overdensity threshold $\mathcal{D}$ for star formation to take place. On the other 
Table 2. Collection of cosmological models, simulation specifics and feedback parameters. The box length $L_{\mathrm{box}}$ is given in $h^{-1} \mathrm{Mpc}$, the cell length $L_{\text {cell }}$ is given in $h^{-1} \mathrm{kpc}$ and the mass of dark matter particles is given in $10^{6} M_{\odot}$.

\begin{tabular}{ccccccccccccc}
\hline \hline EXPERIMENT & $n_{\mathrm{r}}$ & $\Omega_{\mathrm{DM}}$ & $\Omega_{\mathrm{b}}$ & $\Omega_{\Lambda}$ & $h$ & $\sigma_{8}$ & $N$ & $L_{\text {box }}$ & $L_{\text {cell }}$ & $M_{\text {DM }}$ & $A$ & $\mathcal{D}$ \\
\hline & & & & & & & & & & & & \\
$\Lambda$ CDM1 & 1 & 0.325 & 0.025 & 0.65 & 0.7 & 0.9 & $350^{3}$ & 30 & 85.7 & 28.1 & 200 & 100 \\
$\Lambda$ CDM2 & 1 & 0.325 & 0.025 & 0.65 & 0.7 & 0.9 & $270^{3}$ & 21.4 & 79.3 & 22.2 & 200 & 100 \\
$\Lambda$ CDM3 & 1 & 0.325 & 0.025 & 0.65 & 0.7 & 0.9 & $300^{3}$ & 12 & 40 & 2.85 & 200 & 100 \\
$\Lambda$ CDM4 & 9 & 0.37 & 0.03 & 0.6 & 0.6 & 0.9 & $128^{3}$ & 5 & 39.1 & 2.22 & 200 & $100,500,1000$ \\
$\Lambda$ CDM5 & 18 & 0.324 & 0.026 & 0.65 & 0.7 & 0.9 & $128^{3}$ & 5 & 39.1 & 2.65 & $0,50,200$ & 100 \\
SCDM & 18 & 0.95 & 0.05 & 0 & 0.5 & 1.2 & $128^{3}$ & 5 & 39.1 & 3.96 & $0,50,200$ & 100 \\
BSI & 18 & 0.95 & 0.05 & 0 & 0.5 & 0.6 & $128^{3}$ & 5 & 39.1 & 3.96 & $0,50,200$ & 100 \\
\hline
\end{tabular}

hand, supernova explosions can evaporate the cold gas phase and increase the temperature of the hot component. In our model, the supernova feedback parameter $A$ controls the amount of energy (relative to the supernovae mass) that goes into cold cloud evaporation:

$$
\begin{aligned}
& \left(\dot{\rho}_{\mathrm{h}}\right)_{\mathrm{SN}}=-\left(\dot{\rho}_{\mathrm{c}}\right)_{\mathrm{SN}}=\frac{\beta \rho_{\mathrm{c}}}{t_{*}} A \\
& \left(\dot{E}_{\mathrm{h}}\right)_{\mathrm{SN}}=\frac{\beta \rho_{\mathrm{c}}}{t_{*}}\left(\epsilon_{\mathrm{SN}}+A \epsilon_{\mathrm{c}}\right)
\end{aligned}
$$

where the subscripts $\mathrm{h}$ and $\mathrm{c}$ denote hot and cold gas, $\epsilon_{\mathrm{SN}} \simeq 4.45 \times 10^{49} \mathrm{erg} M_{\odot}^{-1}$ is the supernovae specific energy (Salpeter IMF) and $\epsilon_{\mathrm{c}}$ is the specific energy of the cold gas phase $\left(T_{\mathrm{c}}=10^{4} \mathrm{~K}\right)$.

\subsection{Numerical experiments}

Table 2 summarizes our set of experiments. In order to improve statistics, several realizations were run when the number of particles was low enough $\left(N=128^{3}\right)$, changing the random seeds used to generate the initial conditions. The number of realizations is given by $n_{\mathrm{r}}$ in the second column. The cosmological model and the normalization $\sigma_{8}$ are shown in the next 5 columns, as well as the number of particles, box size, cell size (which determines our spatial resolution) and mass of each dark matter particle (mass resolution). In the last two columns we present the values of the supernova feedback parameter $A$ and the overdensity threshold $\mathcal{D}$ required for star formation.

The comparison of experiments $\Lambda$ CDM5, SCDM and BSI allows us to determine the effect of cosmology and feedback from supernovae on the global SFR history. Effects of photoionization are discussed from the results of experiment $\Lambda \mathrm{CDM} 4$, and the dependence on resolution and volume can be studied from the whole set of $\Lambda$ CDM experiments. In $\Lambda$ CDM 1 and 2 , the volume is high enough to include a cluster of galaxies, so we have also investigated the behavior of the SFR in different environments.

\subsection{Resolution effects}

In pure $N$-body simulations the lack of mass and force resolution leads to the well known overmerging problem (see e.g. Klypin et al. 1999; van Kampen 2001), i.e. substructures of large halos or halos inside of halos are erased.

This effect becomes important in galaxy clusters. To avoid overmerging high force and mass resolution are necessary which may be reached later in adaptive mesh and multimass $N$-body/gasdynamical codes.

Our code is based on a fixed Eulerian mesh, so resolution is limited to the cell size. High spatial resolution can only be achieved by increasing the number of cells uniformly, or reducing the computational volume. As a trade off between the two, we have decided to restrict us on medium spatial resolution, in favour of large simulation volumes. Resolution effects are discussed in detail in the next section.

The numerical code has been parallelized using OpenMP compiler directives. Therefore, it runs very efficiently in parallel computers with either Shared or Non Uniform Memory Access (NUMA) architectures. The simulations have been performed on a variety of machines.

\section{Results}

\subsection{Cosmology dependence}

The global star formation histories from simulations of the three cosmological scenarios considered are compared in Fig. 2. The initial power spectrum normalization determines the evolution of structures, which represent a crucial factor in determining the rate of conversion of gas to stars.

Cosmological scenarios with CDM alone cannot explain structure formation on both small and very large scales. However, we have included SCDM as a reference model in our comparison. Due to its large power at small scales, SCDM produces many structures at high redshift. Therefore, we get a flat star formation rate even beyond $z \sim 6$, with a plateau about one decade over the observations (Fig. 2, middle panel). It does not seem very likely that this difference can be explained by dust absorption. In this model, too many baryons are transformed into stars too early with respect to observations, which poses an additional problem for the SCDM model.

On the contrary, the BSI model leads to a clear maximum in the SFR at $z \sim 2$, but the absolute value is too small and the decrease at higher redshifts is clearly too 


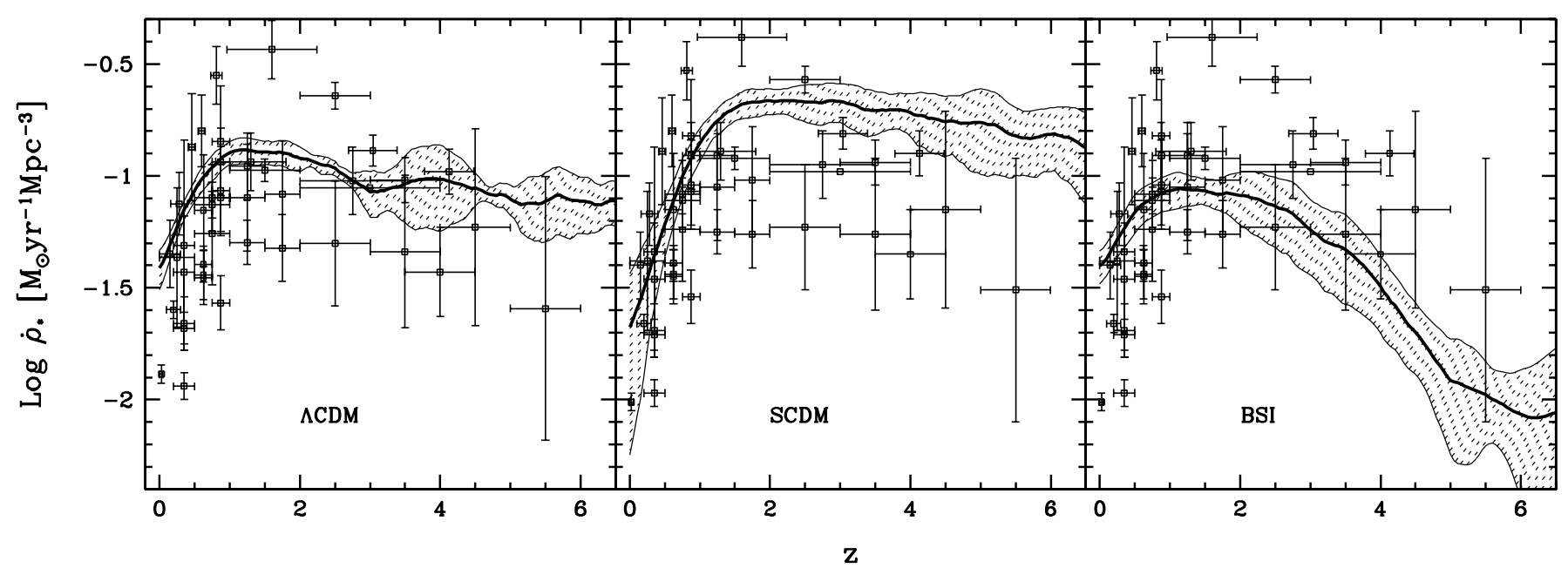

Fig. 2. Evolution of the comoving SFR density in different cosmologies (experiments $\Lambda$ CDM5, SCDM and BSI). The average value over different realizations is plotted as a thick solid line, while the shaded area represents the $1 \sigma$ deviation from the mean. Dots correspond to the observational data points listed in Table 1.

steep (Fig. 2, right). This model has not enough power at small scales for producing enough stars at high redshift. As a side effect, the cosmic star formation rate density remains too high at low redshifts, since a large part of cold gas remains in high density regions triggering further star formation activity.

A cosmological model with non-zero cosmological constant offers the best agreement with observations (Fig. 2, left). Both the overall trend and the height of the plateau fit reasonably well to the observational data points. At present, the $\Lambda$ CDM model fits best all known observational data (e.g. Bahcall et al. 1999). Our results confirm that this model can also reproduce the observed star formation history of the universe when dust extinction is taken into account, in which case a significant amount of star formation is expected to take place at high redshift.

A further feature (common to all cosmologies) is the smooth redshift dependence of the mean SFR and the small range of scatter. This is in sharp contrast to the evolution of individual baryonic clumps, or the behavior of different regions in a single time-step of the simulation. There we observe huge bursts of star formation with an increase in activity by a factor of 10 and higher, as well as a quiescent regime with a star formation rate lower than the average.

In these simulations, the first stars are created around $z \sim 10$, which seems to be not too unrealistic, but it may depend on resolution. This was not a primary issue of the present study.

\subsection{Feedback dependence}

Figure 3 shows the results of experiments $\Lambda$ CDM5 and $\Lambda \mathrm{CDM} 4$, from where the effects of supernova feedback and photoionization were studied. The same trends described in this section were also seen in SCDM and BSI simulations.
Supernovae (left panel of Fig. 3) play a key role in the slope of the SFR density at low $z$. Gas heating and evaporation act as a self-regulating mechanism that inhibits subsequent gas infall when many new stars are formed. This is specially important in the most massive objects, which show a much higher star formation activity when feedback is not implemented. Supernova explosions can also blow away the gas reservoirs of dwarf galaxies. The effect on the global star formation rate is more noticeable at high redshift, when there are still very few galaxies massive enough to retain the heated gas within their gravitational potential wells. However, photoionization (right panel) is the dominant effect at very early epochs, preventing the gas from cooling and forming stars in low density regions (Efstathiou 1992). As we have discussed above, our model takes into account this effect through the overdensity threshold parameter $\mathcal{D}$ for star formation. Only in regions denser than $\mathcal{D}$, molecular clouds will be able to form, and the onset of cosmic star formation activity is

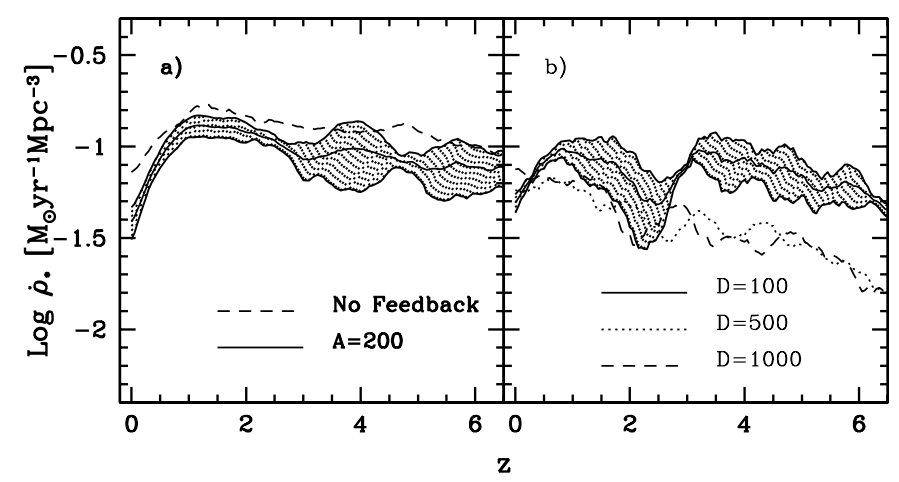

Fig. 3. Effects of stellar feedback on the cosmic SFR density. a) $\Lambda$ CDM5 simulations with supernova feedback parameter $A=200$ (solid line) within $1 \sigma$ error (shaded area) and $A=0$ (dashed line). b) Same as a) but for $\Lambda$ CDM4 simulations run with different values of the overdensity threshold $\mathcal{D}$ imposed by photoionization. 
thus retarded until more and more objects become dense enough to screen the photoionizing background.

We found a SFR density consistent with observational data for $\mathcal{D} \sim 100$. This is in roughly agreement with results from a more detailed theoretical estimate of the same parameter (Mücket \& Kates 1997), although in that case the actual value of $\mathcal{D}$, as well as the temperature range in which thermal instability is an efficient process, depend on the local gas density and the intensity of the ionizing flux.

\subsection{Environmental dependence}

In the hierarchical scenario of structure formation, the first objects collapsed around the highest overdensity peaks and merged to eventually form the giant ellipticals in the centers of clusters and the bulges of the most massive isolated galaxies. These objects contain the vast majority of stars older than $z=3$, and hence their star formation history must be completely different from that of galaxies living in less dense environments.

The experiments $\Lambda$ CDM 1 and 2 sample a volume containing clusters, filaments, and groups of galaxies as well as isolated galaxies. Gottlöber et al. (2001) have studied the merging history of dark matter halos as function of halo environment. They found that halos located inside clusters have formed earlier than isolated halos of the same mass. Moreover, they showed that at higher redshifts $(z \sim 1-4)$, progenitors of cluster and group galaxies have 3-5 times higher merger rates than isolated galaxies. Mergers of galaxies are thought to play a crucial role in the evolution of galaxies. In particular, the inflow of material may serve as a source of fresh gas and therefore increase the star formation rate.

In Fig. 4 we plot the contribution to the cosmic SFR density of progenitors of objects more massive than $10^{13} M_{\odot}$ at present (clusters, large groups) and less massive (isolated galaxies or small groups). The most massive objects have their peaks of star formation activity around $z \sim 3$, showing passive evolution since $z \sim 2$. This is in excellent agreement with the merging history found by Gottlöber et al. (2001). The progenitors of these objects were responsible for the bulk of star formation at high redshift, and can be very likely associated with the Lyman Break Galaxy population.

Isolated galaxies and galaxies in small groups are younger. They are responsible for most of the current stellar production. In these areas, halos massive enough to retain their gas content have a nearly constant SFR (with sporadic burst episodes).

The simulated volume in our numerical experiments is too small to be a representative cosmological volume. In fact, the larger volume $\Lambda$ CDM1 contains only one galaxy cluster of approximately $3 \times 10^{14} M_{\odot}$ whereas the samller volume $\Lambda$ CDM2 contains four massive groups of about $5 \times 10^{13} M_{\odot}$. Comparing the solid lines in Fig. 4 we find qualitatively the expected scenario: the peak of star

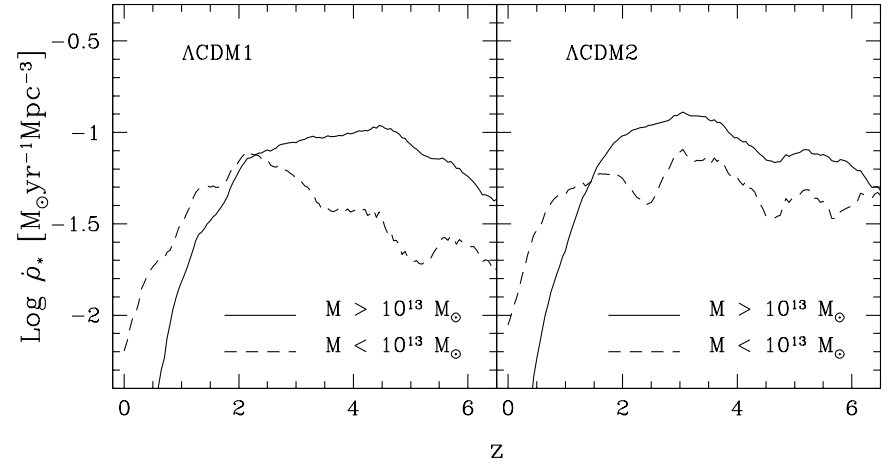

Fig. 4. Star formation rate densities in models $\Lambda$ CDM1 and $\Lambda \mathrm{CDM} 2$ due to halos more (solid lines) and less (dashed lines) massive than $10^{13} M_{\odot}$ at $z=0$.

formation in the progenitors of the more massive cluster happened earlier (about $z=4.5$ ) than for the less massive groups $(z=3)$.

In the absence of stellar feedback, most of the baryonic matter would have cooled into small mini-galaxies (Cole 1991; White \& Frenk 1991; Blanchard et al. 1992). Photoionization and supernova explosions inhibit cooling and star formation in very low-mass objects, so early star formation was biased towards the high overdensity regions that will collapse later to form the cores of the first clusters.

As the collapse proceeds, gas is heated to temperatures that make the cooling times too long and the SFR drastically drops once the cold gas has been exhausted.

At the same time, new galaxies will form and produce stars. Some of them will remain in rather isolated areas until present (dashed lines in Fig. 4) and some others will fall into the potential wells of clusters. It seems very likely that tidal interactions trigger intense star formation bursts in these infalling galaxies (Lavery \& Henry 1988), but ram pressure of the ICM may also deprive them from their cold gas reservoirs (Gunn \& Gott 1972; Quilis et al. 2000). This later mechanism is supported by the observational evidence of the displacement of the neutral hydrogen disk of poststarburst galaxies with respect to the stellar population (Valluri \& Jog 1991).

Cluster galaxies would then stop/decay their star formation activity and become redder, giving rise to the Butcher \& Oemler (1978) effect. At the same time, they will experience a morphological transformation, due to mergers with their neighboring cluster galaxies, from spirals into S0 (Dressler et al. 1997). At present, most of the observed clusters are at $z \leq 0.5$ (Fasano et al. 2000, and references therein), so deeper surveys are clearly needed in order to check this scenario.

\subsection{Resolution and volume dependence}

Star formation and feedback processes are incorporated in gasdynamical simulations (either $\mathrm{SPH}$ or Eulerian) by means of rather simple recipes which pretend to 


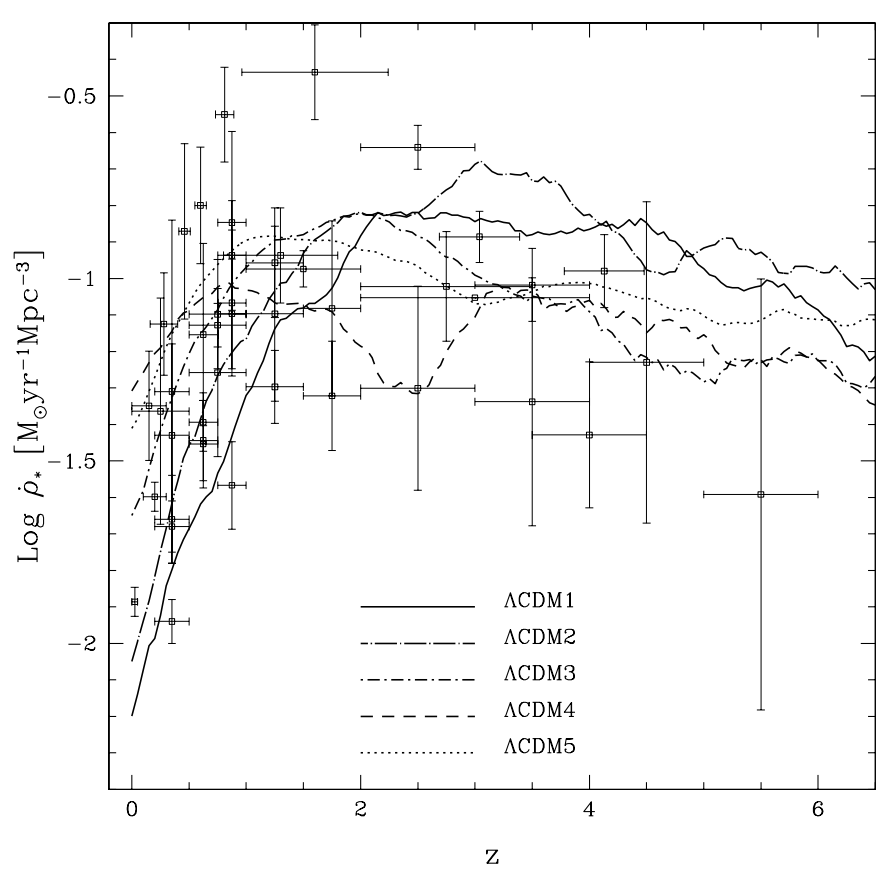

Fig. 5. Comoving SFR in our $5 \Lambda \mathrm{CDM}$ experiments, all with $A=200$ and $\mathcal{D}=100$. A significant star formation activity is found at high $z$ regardless of resolution and volume. This is consistent with the full set of observational data points (Table 1).

extrapolate the effects of local physics acting on scales of a few pc and masses of $\sim 10^{5} M_{\odot}$ below the resolution limit (often at kpc and higher). In our case, part of this extrapolation is hidden in the two parameters: The supernova feedback parameter $A$ and the overdensity threshold for star formation $\mathcal{D}$. Whether this parameterization is a reasonable extrapolation of the underlying subgrid physics depends on the stability of results against changes in spatial resolution of the simulations.

On the other hand, when one tries to estimate cosmological averaged quantities, like the SFR density, it is necessary to check for deviations of these quantities due to small number statistics. In the case that matters here, a volume averaged quantity, the cosmic variance can have a relatively large contribution in the particular determination of the comoving SFR density. Thus, we have simulated different computational volumes with similar resolutions, depending on the computational facilities.

To check for these two effects we compare in Fig. 5 our results for $\Lambda \mathrm{CDM}$ simulations with different resolutions and volumes.

The most noticeable feature that we derive from this figure is the steeper slope of the local SFR for larger volume simulations. As we increase the volume, larger structures are being simulated (i.e. clusters) and as explained above, the reduced star formation activity in the cores of clusters in recent epochs can explain this behavior. In simulations with smaller volumes, most of the galaxies that form are either isolated or in small groups, which have a completely different star formation activity. Thus, we can conclude from our simulations that the effects of cosmic variance are quite important in determining the proper behavior of the cosmic SFR density from $z \geq 1$ to the present.

We do not find significant differences in our estimates of the SFR density from simulations with different volumes and spatial resolutions, but there is a general trend to have more SFR density at early times for simulations with higher resolution. This is a consequence of the overcooling problem (see Balogh et al. 2001 for a recent review), since the number of resolved low mass halos increases in these simulations. A detailed analysis of this question would require a more realistic treatment of photoionization.

Keeping all these considerations in mind, we should be extremely cautious when making quantitative estimates of the cosmic SFR density at any given $z$. However, this quantity shows a similar behavior in all $\Lambda \mathrm{CDM}$ experiments. This is a very promising result indicating that its qualitative evolution (particularly at high redshift) is a robust prediction of our simulations.

No characteristic epoch of cosmic star formation is found for a $\Lambda$ CDM universe. The comoving SFR density increases with lookback time until it reaches a nearly constant mean plateau beyond $z=2$. Stars probably formed gradually at high redshift, according to most recent observations and taking dust extinction into account. At some point around $z \sim 1$, the global star formation activity would have declined sharply until the present day, although individual SFRs of active galaxies are kept approximately constant.

The star density $\rho_{*}(z)$ shows a similar behavior for both CDM and $\Lambda$ CDM models. The most remarkable difference is found in the present value of $\rho_{*}(0)$ (see Table 3 ), which can be observationally deduced from the local luminosity density, assuming a constant mass-to-light ratio (Madau et al. 1996; Fukugita et al. 1998):

$\rho_{*}^{\mathrm{obs}}(0) \sim 7 \times 10^{8} h M_{\odot} \mathrm{Mpc}^{-3}$

Based on the fraction of stars in bulges, ellipticals and S0s, Renzini (1998) estimated that about $30 \%$ of the stellar content of the universe should have already been formed by $z=3$, regardless of the cosmological model. The amount of stars produced in experiments $\Lambda$ CDM1 and 2 (Table 3 ), both at $z=3$ and $z=0$, are in fair agreement with these observationally derived quantities. For the other experiments, the fraction of stars older than $z=3$ is significantly lower, which is not only due to a low star formation activity at high redshift, but also to a higher SFR in the local universe and the effects of cosmic variance due to the smaller simulated volumes.

\section{Conclusions}

We have performed 66 numerical simulations, grouped in 7 different experiments, in order to obtain a theoretical prediction of the cosmic star formation history. We compared our predictions with a number of published 
Table 3. Present star density (in $10^{8} h M_{\odot} \mathrm{Mpc}^{-3}$ ) and fraction of stars older than $z=3$ for the different numerical experiments.

\begin{tabular}{ccc}
\hline \hline EXPERIMENT & $\rho_{*}(z=0)$ & $\rho_{*}(z=3) / \rho_{*}(0)$ \\
\hline$\Lambda$ CDM1 & 6.50 & 0.252 \\
$\Lambda$ CDM2 & 8.60 & 0.243 \\
$\Lambda$ CDM3 & 9.51 & 0.115 \\
$\Lambda$ CDM4 & 9.01 & 0.116 \\
$\Lambda$ CDM5 & 10.9 & 0.119 \\
SCDM & 12.3 & 0.115 \\
BSI & 7.37 & 0.041 \\
\hline
\end{tabular}

observational estimates. We summarize our results as follows:

1. $\Lambda$ CDM shows the best agreement with observations. Standard CDM tends to overpredict the cosmic SFR density, while BSI fails to form enough stars at high $z$ due to its reduced power at small scales.

2. Photoionization fixes the onset of star formation in the universe, supressing star formation in low-mass objects at high $z$. Supernovae feedbacks are very efficient to self-regulate the process of converting gas into stars, preventing the most massive halos to form too many stars at later epochs.

3. Star formation histories are very different for objects that end up in the cores of clusters and isolated galaxies. Galaxies in cluster cores have much older stellar populations, and their activity is drastically reduced when they lose their cold gas reservoirs.

4. In contrast to the first observational estimates (Madau et al. 1996), but in agreement with most recent data (Steidel et al. 1999), the $\Lambda$ CDM model predicts almost no drop in the cosmic SFR for $2<z<5$. Star formation seems to be a gradual process with no characteristic epoch.

5. The comoving simulated volumes in our experiments are comparable to those covered by observations. Thus, one should expect a non negligible error in the observational measurements due to cosmic variance associated with small volume statistics. This is most important when one wants to determine the steep slope of the SFR density from $z \sim 1$ to the present time.

Selection effects, dust extinction and the conversion from luminosities to SFRs are the major sources of uncertainty in the observational estimates. The small volume sampled by the HDF may also introduce some statistical bias at high redshift.

Uncertainty in our numerical experiments comes from spatial resolution and the star formation and feedback prescriptions. The use of Adaptive Mesh Refinement Eulerian codes (AMR) will be useful in order to improve resolution in statistically significant cosmological volumes. Photoionization and chemical enrichment have been given a phenomenological treatment in our code. It would be extremely interesting to self-consistently model both processes, computing the intensity of the ionizing background at each time-step, as well as metal production and advection.

Acknowledgements. This work has been partially supported by the SEUID (Spain) under project number PB96-0029, by the Acciones Integradas Hispano-Alemanas HA2000-0026 and by Deutscher Akademischer Austauschdienst DAAD.

We thank the Centro Europeo de Paralelismo de Barcelona and the Centro de Computación Científica de la Universidad Autónoma de Madrid for allowing us to use their computational facilities in which most of the simulations reported in this paper were performed.

\section{References}

Bahcall, N. A., Ostriker, J. P., Perlmutter, S., \& Steinhardt, P. J. 1999, Science, 284, 1481

Balogh, M. L., Pearce, F. R., Bower, R. G., \& Kay, S. T. 2001, MNRAS, 326, 1228

Blanchard, A., Valls-Gabaud, D., \& Mamon, G. A. 1992, A\&A, 264, 365

Butcher, H., \& Oemler, A. 1978, ApJ, 226, 559

Cole, S. 1991, ApJ, 367, 45

Cole, S., Aragon-Salamanca, A., Frenk, C. S., Navarro, J. F., \& Zepf, S. E. 1994, MNRAS, 271, 781

Connolly, A. J., Szalay, A. S., Dickinson, M., Subbarao, M. U., \& Brunner, R. J. 1997, ApJ, 486, L11

Cowie, L. L., Songaila, A., \& Barger, A. J. 1999, AJ, 118, 603

Dressler, A., Oemler, A., Couch, W. J., et al. 1997, ApJ, 490, 577

Efstathiou, G. 1992, MNRAS, 256, 43P

Efstathiou, G., Bond, J. R., \& White, S. D. M. 1992, MNRAS, $258,1 \mathrm{P}$

Elizondo, D., Yepes, G., Kates, R., \& Klypin, A. 1999, New Astron., 4, 101

Elizondo, D., Yepes, G., Kates, R., Müller, V., \& Klypin, A. 1999, ApJ, 515, 525

Fasano, G., Poggianti, B. M., Couch, W. J., et al. 2000, ApJ, 542,673

Flores, H., Hammer, F., Thuan, T. X., et al. 1999, ApJ, 517, 148

Fukugita, M., Hogan, C. J., \& Peebles, P. J. E. 1998, ApJ, 503, 518

Gallego, J., Zamorano, J., Aragon-Salamanca, A., \& Rego, M. 1995, ApJ, 455, L1

Gispert, R., Lagache, G., \& Puget, J. L. 2000, A\&A, 360, 1

Giroux, M., \& Shapiro, P. 1986, ApJS, 102, 191

Glazebrook, K., Blake, C., Economou, F., Lilly, S., \& Colless, M. 1999, MNRAS, 306, 843

Gottlöber, S., Müller, V., \& Starobinsky, A. A. 1991, Phys. Rev. D, 43, 2510

Gottlöber, S., Klypin, A., \& Kravtsov, A. V. 2001, ApJ, 546, 223

Gunn, J. E., \& Gott, J. R. I. 1972, ApJ, 176, 1

Haarsma, D. B., Partridge, R. B., Windhorst, R. A., \& Richards, E. A. 2000, ApJ, 544, 641

Hammer, F., Flores, H., Lilly, S. J., et al. 1997, ApJ, 481, 49

Hopkins, A. M., Connolly, A. J., Haarsma, D. B., \& Cram, L. E. 2001, AJ, 122, 288

Hughes, D. H., Serjeant, S., Dunlop, J., et al. 1998, Nature, 394, 241

van Kampen, E. 2001, MNRAS, submitted [astro-ph/0008453] 
Kauffmann, G., White, S. D. M., \& Guiderdoni, B. 1993, MNRAS, 264, 201

Kennicutt, R. C. 1998, ARA\&A, 36, 189

Klypin, A., Gottlöber, S., Kravtsov, A. V., \& Khokhlov, A. M. 1999, ApJ, 516, 530

Lavery, R. J., \& Henry, J. P. 1988, ApJ, 330, 596

Lilly, S. J., Le Fevre, O., Hammer, F., \& Crampton, D. 1996, ApJ, 460, L1

Madau, P., Ferguson, H. C., Dickinson, M. E., et al. 1996, MNRAS, 283, 1388

Madau, P., Pozzetti, L., \& Dickinson, M. 1998, ApJ, 498, 106

Massarotti, M., Iovino, A., \& Buzzoni, A. 2001, ApJ, 559, L105

Melchiorri, A., Ade, P. A. R., de Bernardis, P., et al. 2000, ApJ, 536, L63

Mücket, J. P., \& Kates, R. E. 1997, A\&A, 324, 1

Nagamine, K., Fukugita, M., Cen, R., \& Ostriker, J. P. 2001, ApJ, 558, 497

Pascarelle, S. M., Lanzetta, K. M., \& Fernández-Soto, A. 1998, ApJ, 508, L1

Perlmutter, S., Aldering, G., Goldhaber, G., et al. 1999, ApJ, 517,565

Pettini, M., Kellogg, M., Steidel, C. C., et al. 1998, ApJ, 508, 539

Quilis, V., Moore, B., \& Bower, R. 2000, Science, 288, 1617
Renzini, A. 1998, in The Young Universe: Galaxy Formation and Evolution at Intermediate and High Redshift, ASP Conf. Ser., 146, 298

Sawicki, M. J., Lin, H., \& Yee, H. K. C. 1997, AJ, 113, 1

Schmidt, M. 1968, ApJ, 151, 393

Somerville, R. S., \& Primack, J. R. 1999, MNRAS, 310, 1087

Somerville, R. S., Primack, J. R., \& Faber, S. M. 2001, MNRAS, 320, 504

Steidel, C. C., Giavalisco, M., Pettini, M., Dickinson, M., \& Adelberger, K. L. 1996, ApJ, 462, L17

Steidel, C. C., Giavalisco, M., Dickinson, M., \& Adelberger, K. L. 1996, AJ, 112, 352

Steidel, C. C., Adelberger, K. L., Giavalisco, M., Dickinson, M., \& Pettini, M. 1999, ApJ, 519, 1

Sutherland, R. S., \& Dopita, M. A. 1993, ApJS, 88, 253

Tresse, L., \& Maddox, S. J. 1998, ApJ, 495, 691

Treyer, M. A., Ellis, R. S., Milliard, B., Donas, J., \& Bridges, T. J. 1998, MNRAS, 300, 303

Valluri, M., \& Jog, C. J. 1991, ApJ, 374, 103

White, S. D. M., \& Frenk, C. S. 1991, ApJ, 379, 52

Yan, L., McCarthy, P. J., Freudling, W., et al. 1999, ApJ, 519, $\mathrm{L} 47$

Yepes, G., Kates, R., Khokhlov, A., \& Klypin, A. 1997, MNRAS, 284, 235 